\title{
PRODUCTION AND INNOVATIVE APPLICATIONS OF CRYOGENIC SOLID PELLETS*
}

\author{
By \\ M. J. Gouge, L. R. Baylor, S. K. Combs, P. W. Fisher, C. A. Foster, \\ C. R. Foust, and S. L. Milora \\ Oak Ridge National Laboratory
}

\author{
Presented at the \\ Cryogenic Engineering Conference/ \\ International Cryogenic Materials Conference \\ CEC/ICMC 1999 Symposium \\ Montreal, Quebec, Canada \\ Palais des Congres
}

July 12, 1999

\author{
Prepared at the \\ Oak Ridge National Laboratory \\ Post Office Box 2009 \\ Oak Ridge, TN 37831-8071 USA \\ Managed by \\ LOCKHEED MARTIN ENERGY RESEARCH CORP. \\ for the \\ U.S. DEPARTMENT OF ENERGY \\ under contract DE-AC05-960R22464
}

\footnotetext{
*Research at Oak Ridge National Laboratory was sponsored by the Office of Fusion Energy Sciences for the U.S. Department of Energy under contract DE-AC05-96OR22464 with Lockheed Martin Energy Research Corp.
} 



\title{
PRODUCTION AND INNOVATIVE APPLICATIONS OF CRYOGENIC SOLID PELLETS
}

\author{
M. J. Gouge, L. R. Baylor, S. K. Combs, P. W. Fisher, \\ C. A. Foster, C. R. Foust, and S. L. Milora \\ Oak Ridge National Laboratory \\ P.O. Box 2009, Oak Ridge, TN 37831-8071, USA
}

\begin{abstract}
For over two decades Oak Ridge National Laboratory has been developing cryogenic pellet injectors for fueling hot, magnetic fusion plasmas. Cryogenic solid pellets of all three hydrogen isotopes have been produced in a size range of 1- to 10 -mm diameter and accelerated to speeds from $<100$ to $\sim 3000 \mathrm{~m} / \mathrm{s}$. The pellets have been formed discretely by cryocondensation in gun barrels and also by extrusion of cryogenic solids at mass flow rates up to $\sim 0.26 \mathrm{~g} / \mathrm{s}$ and production rates up to ten pellets per second. The pellets traverse the hot plasma in a fraction of a millisecond and continuously ablate, providing fresh hydrogenic fuel to the interior of the plasma. From this initial application, uses of this technology have expanded to include (1) cryogenic xenon drops or solids for use as a debris-less target in a laser plasma source of X-rays for advanced lithography systems, (2) solid argon and carbon dioxide pellets for surface cleaning or decontamination, and (3) methane pellets in a liquid hydrogen bath for use as an innovative moderator of cold neutrons. Methods of production and acceleration/transport of these cryogenic solids will be described, and examples will be given of their use in prototype systems.
\end{abstract}

\section{INTRODUCTION}

Oak Ridge National Laboratory (ORNL) has been developing cryogenic pellet systems for over two decades with a primary emphasis on production and acceleration of solid pellets of the three hydrogen isotopes for fueling the interior of hot plasmas. Building on this technology, other applications of cryogenic pellets have been developed to the prototype stage, including (1) cryogenic xenon drops or solids for use as a debris-less target in a laser plasma X-ray source, (2) solid argon and carbon dioxide pellets for surface cleaning or decontamination, and (3) methane pellets in a liquid hydrogen bath for use as a cold neutron moderator. Each of these innovative applications of cryogenic pellets will be described in more detail. 


\section{HYDROGENIC PELLETS FOR PLASMA FUELING}

It was recognized early in the magnetic fusion development program that fueling of hot plasmas by the traditional method of puffing hydrogenic $\left(\mathrm{H}_{2}, \mathrm{D}_{2}, \mathrm{~T}_{2}\right)$ gas into the vacuum vessel would be problematic because the gas molecules do not penetrate far into the plasma core where they are needed to replenish hydrogenic ions consumed by the deuterium-tritium (D-T) or deuterium-deuterium (D-D) fusion reactions. An alternate method of fueling was proposed where solid pellets of all three hydrogen isotopes are injected into the plasma at high speeds and continuously ablate, providing fuel to the plasma interior. For reference, relevant properties of the hydrogen isotopes are provided in Table 1. Due to the low boiling points, typically liquid helium or cold gaseous helium has been used as the cryogenic fluid to cool and solidify the hydrogenic feed gas, although more recently Gifford-McMahon (GM) cryocoolers have been used for discrete pellet production. For radioactive tritium pellets, the cryogenic cooling also has to accommodate the volume heat source caused by the beta decay of about $1 \mathrm{~W} /$ mole.

There has been much progress in the physics and technology of pellet fueling since this technique was first applied to magnetic fusion experiments over 20 years ago. ${ }^{1,2}$ From first experiments with a single, small hydrogen pellet at hundreds of meters per second, the technology has advanced to hydrogen/deuterium/tritium $(\mathrm{H} / \mathrm{D} / \mathrm{T})$ pellets in longer pulse length systems, with inventories up to about 1000 pellets and speeds in the kilometer-persecond range. Table 2 below provides a summary of the progress of this technology.

Pellets are typically accelerated by pneumatic (light gas gun) injectors using hydrogen or helium propellant gas or via centrifuge acceleration. ${ }^{2}$ The low density of the hydrogenic solids as noted in Table 1 allows respectable speeds of 1-2 km/s with single-stage light gas guns with propellant gas pressures on the order of 10-100 bar. Two-stage light gas guns operate repetitively at $1 \mathrm{~Hz}$ with 3 -mm pellets and speeds in the range of 2.5 to $3 \mathrm{~km} / \mathrm{s}$. Single 4-mm-diam pellets have been accelerated to speeds above $4 \mathrm{~km} / \mathrm{s}$ with two-stage guns. Centrifuges have accelerated 1 - to $4-\mathrm{mm}$ pellets to speeds over $1 \mathrm{~km} / \mathrm{s}$.

Hydrogenic pellets have been produced discretely in-situ ${ }^{2}$ by admitting feed gas into the interior of a barrel in which a short section (a few millimeters) is cooled to $\sim 6-12 \mathrm{~K}$ by a helium-cooled, copper block attached to the outer diameter of the barrel. Alternatively, a GM cryocooler could cool the copper block to 8-12 K. The feed gas forms a cylindrical solid pellet inside the gun barrel; typical dimensions are a few to $10 \mathrm{~mm}$ diameter and aspect ratios (length to diameter) from 1-2. Another method of hydrogen ice production is

Table 1. Properties of hydrogen isotopes

\begin{tabular}{lccc}
\hline Property & Hydrogen & Deuterium & Tritium \\
\hline Density, g/cm & & 0.2 & 0.32 \\
Boiling point at $1 \mathrm{~atm}, \mathrm{~K}$ & 0.09 & 23.7 & 25 \\
Triple point, K & 20.4 & 18.7 & 20.6 \\
Triple point pressure, torr & 54.8 & 129 & 162 \\
Shear strength, MPa & $0.1(10 \mathrm{~K})$ & $0.5(9 \mathrm{~K})$ & $1.2(9 \mathrm{~K})$ \\
\hline
\end{tabular}

Table 2. Progress in pellet technology 1978-1999

\begin{tabular}{lll}
\hline Parameter & Status 1978 & Status 1999 \\
\hline Pellet isotope & Hydrogen & H/D/T \\
Pellet size & One to a few millimeters & $0.5-10 \mathrm{~mm}$ \\
Pellet inventory & One to few & $1-1200$ \\
Pellet feed rate & N/A & $0.26 \mathrm{~g} / \mathrm{s}$ \\
Pellet speed & Hundreds of meters per second & Thousands of meters per second \\
Reliability & Unknown & Sufficient \\
\hline
\end{tabular}


by extrusion. ${ }^{2}$ Extruders are generally capable of moving solid material at a sufficiently high rate of speed to provide a "real time" pellet feed system. The extruder section (see Fig. 1) consists of a liquid reservoir positioned above a cylindrical freezing chamber, which is fitted at its outlet with a copper extrusion assembly including the nozzle. A motor-driven (electric or pneumatic) screw press activates a polyimide piston that moves vertically inside the cylindrical bore of the freezing chamber where the solid hydrogenic charge is located. The extruder assembly has two cryostats, each made of a block of oxygen-free, highconductivity (OFHC) copper that has square cooling channels with a spiral pitch machined into it. A $\sim 25-$ to $50-\mathrm{W}$ nichrome heater is wrapped around and epoxied to the perimeter of each cryostat for cryogenic temperature control. The extrusion nozzle assembly, which terminates just above the pellet feed mechanism position, provides a smooth transition from the cylindrical extruder bore to a smaller cross section nozzle; this area determines the size of the accelerated pellet. Present deuterium extruders typically operate at $\sim 14 \mathrm{~K}$ where the shear strength of the ice is sufficiently low to prevent excessive piston forces at high extrusion rates. In recent experiments at the Tritium Systems Test Assembly facility at Los Alamos National Laboratory, the Tritium Proof-of-Principle Phase II (TPOP-II) pellet injector has produced good quality tritium and D-T extrusions at temperatures in the range 13-14 K (Ref. 3).

The TPOP-II injector will be described in more detail because it has produced tritium and D-T pellets of typical dimensions $(8-10 \mathrm{~mm})$ required for refueling of a fusion reactor. ${ }^{4}$ Figure 2 is a sketch of the TPOP-II repeating pneumatic injector. An extruder assembly similar to that shown in Fig. 1 is housed in a guard vacuum chamber, which provides vacuum insulation of the cold extruder components. Principal components include the extruder mechanism with two copper cryostats, the pellet cutting and chambering mechanism with adjacent copper cryostat, the $\sim 8$-mm-ID gun barrel, and the ${ }^{3} \mathrm{He}$ separator that removes the ${ }^{3} \mathrm{He}$ by-product from the tritium radioactive decay from the incoming tritium supply gas stream. The extruder mechanism freezes input gas into a column of hydrogenic solid in a batch process for use at a later time. As the extruder feeds a continuous supply of solid deuterium (or tritium) to the gun assembly, a reciprocating, electromagnetically driven, breech-side cutting mechanism (punch tube) forms and chambers cylindrical pellets from the extrusion. The chambering mechanism contains a single cryostat, the main body of which is also a block of OFHC.

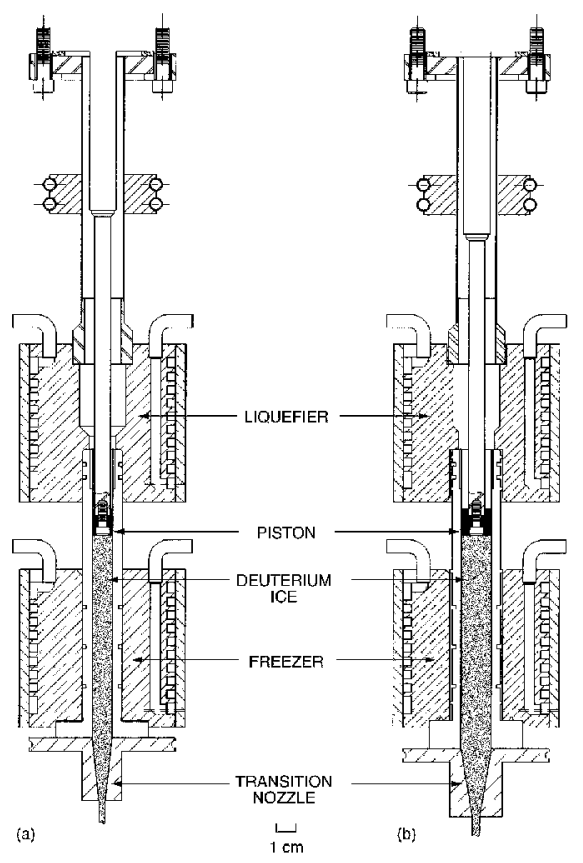

Figure 1. ORNL hydrogenic extruders. 


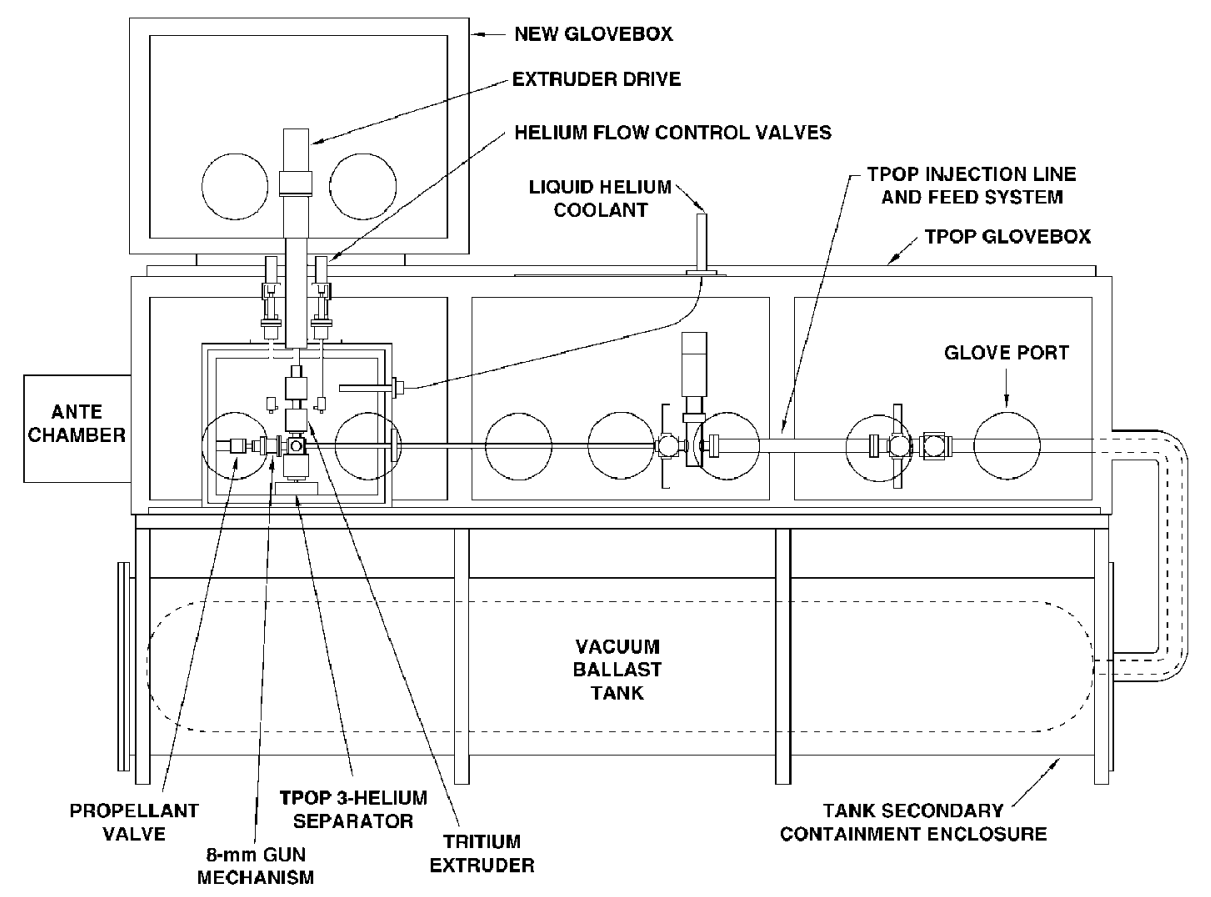

Figure 2. TPOP-II pellet injector.

Tritium and D-T pellets are accelerated in the gun barrel to high speeds with controlled amounts of compressed deuterium gas delivered by a fast electromagnetic propellant valve. This valve is a fast solenoid valve $(<1-\mathrm{ms}$ opening time) developed by ORNL. The gun barrel is a continuous $8-\mathrm{mm}-\mathrm{ID}, 1.25-\mathrm{m}$-long, stainless steel tube with a wall thickness of $0.89 \mathrm{~mm}$. Liquid helium enters and exits the guard vacuum chamber through two vacuum-insulated, O-ring sealed, bayonet fittings (Linde-type) on the front plate. The liquid helium supply is split into four parallel cooling paths inside the guard vacuum box (the two extruder cryostats, the gun cryostat, and the ${ }^{3} \mathrm{He}$ separator), each with a flow control valve. These four parallel paths finally combine and are then discharged through the exhaust bayonet fitting. The ${ }^{3} \mathrm{He}$ separator is basically a small copper counterflow heat exchanger. Helium coolant passes through the central cooling channel, and tritium is cryopumped into the external finned surfaces. D-T pellets up to $10 \mathrm{~mm}$ in size (see Fig. 3) have been extruded at rates up to $0.26 \mathrm{~g} / \mathrm{s}$ for short pulses only; this pellet size and feed rate is sufficient for fueling fusion reactors at the gigawatt power level. 4

The cryogenic pellet injector technology development described previously has resulted in a pneumatic or centrifuge pellet fueling system on virtually every major magnetic fusion experiment in the past two decades. ${ }^{1}$
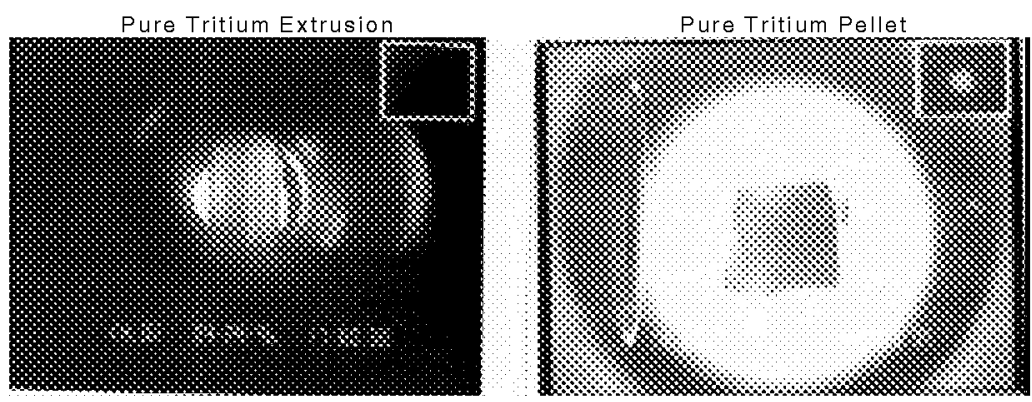

Figure 3. Pure tritium extrusion and pellet. 


\section{XENON DROPLET GENERATOR FOR USE IN A COMPACT LASER PLASMA X-RAY SOURCE}

Soft X-ray projection and proximity lithography systems are being developed for the advanced semiconductor industry for high-resolution feature size printing. A key element in a commercial lithography system would be a compact plasma X-ray source. Compact systems have been developed using a solid state or excimer laser beam impacting a rotating metallic (typically gold or copper) target as a plasma source. ${ }^{5}$ Debris formation and subsequent transport result in impurity deposition or impact damage to process optical components. To mitigate fragmentation damage in a continuous (repetitive) plasma source, discrete droplets delivered at low-to-moderate repetition rates $(\sim 10-100 \mathrm{~Hz})$ are desired. The droplets should be small (mass limited, about $10-50 \mu \mathrm{m}$ in diameter) to ensure complete vaporization by the high-power, short-pulse laser beam. The resulting plasma would generate soft X-rays for use in an advanced lithography system.

To achieve still further improvements in debris reduction, a cryogenic droplet target system for use in an advanced laser plasma source for X-ray lithography has been designed, fabricated, and tested at ORNL. ${ }^{6}$ The advantage of the cryogenic droplets is that small fragments and vapor from the laser-droplet interaction can be collected preferentially on a cold surface and pumped away later at room temperature. It was determined that drop-ondemand droplet generators similar to those used for ink jet printing have potential performance requirements meeting the lithography application. The drop-on-demand feature allows control of the lithography process on a drop-to-drop basis, which is attractive due to the inherent interruptions of the semiconductor wafer etching process for indexing and wafer replacement. This also results in the most efficient use of the feed gas and minimizes the recycled gas stream.

A fundamental decision is the choice of fluid for the cryogenic droplet. This choice is largely driven by the potential to produce intense $\mathrm{X}$ rays from the droplet-laser interaction in the wavelength region of interest. For X-ray lithography systems, solid xenon has excellent X-ray conversion efficiencies for wavelengths in the range of 1-14 $\mathrm{nm}$. Xenon was chosen as the initial cryogenic fluid to use in this system due to the $\mathrm{X}$-ray production efficiencies discussed above. An obvious benefit of xenon is that the boiling and triple point temperatures are high enough that liquid nitrogen is a feasible coolant to maintain the xenon liquid at the specified temperature. The fluid properties of liquid xenon present some unique challenges to droplet production. Water is the most common fluid used in droplet production systems. Liquid xenon has about one-third the surface tension, one-fourth the viscosity, and about three times the density of water. In fact, liquid xenon is denser than several light metals such as aluminum. The Weber number is the ratio of the inertial force to the surface tension force; this dimensionless number is a key parameter in drop-ondemand systems as surface tension forces hold the meniscus in the orifice in between dropon-demand cycles. The Weber number for liquid xenon is about 40 times higher than a water droplet of the same size. A schematic of the xenon drop production device with support components is shown in Fig. 4.

The xenon drops are produced on demand by pulsing a piezoelectric disc attached to a rectangular copper block that has a supply reservoir of liquid xenon. The copper block is maintained at $160-175 \mathrm{~K}$ by conduction cooling via copper straps from a copper cold finger of a liquid nitrogen dewar. A signal generator and radio frequency (RF) amplifier provide the $\sim 20$ - to $100-\mathrm{V}$ square wave drive pulse to produce each drop. The droplet generator is housed in a vacuum chamber that is a six-way cross assembly with 10-in. conflat flanges. Windows are installed on four of the six flanges. An MKS pressure monitoring and differential pressure control system (range 0-10 torr) maintains the helium background pressure in the chamber at typical values in the range 900-1100 torr with a 


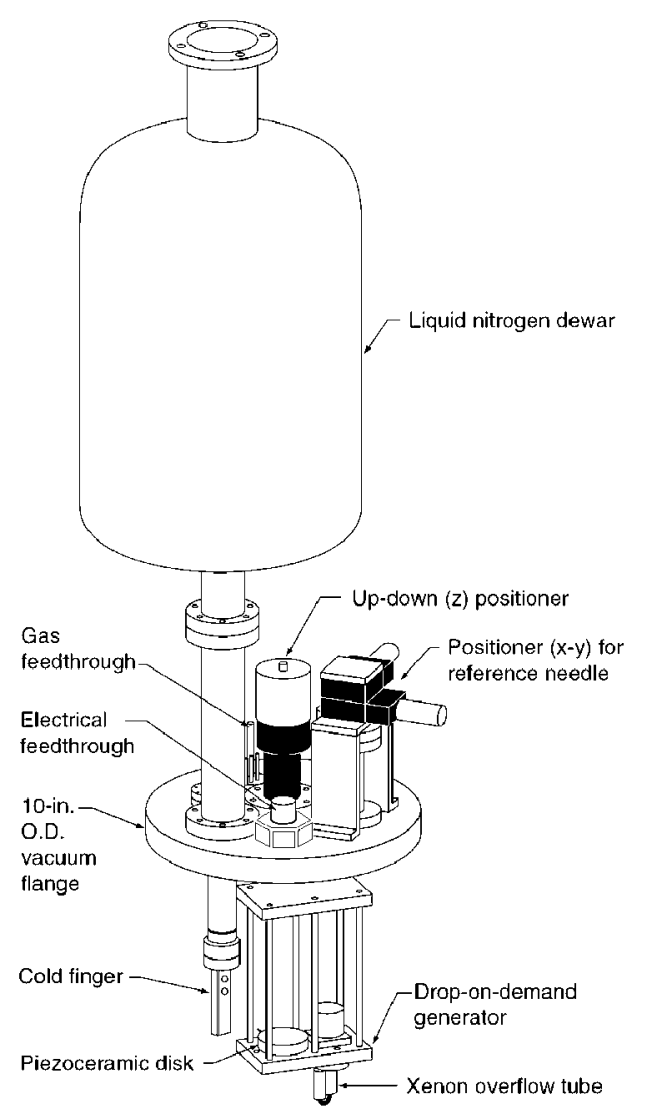

Figure 4. Piezoelectric drop-on-demand generator and support systems.

near-zero differential pressure across the orifice, which has liquid xenon pressure on the interior side and vacuum chamber pressure on the exterior side. Diagnostics include vacuum chamber, helium feed gas and xenon supply gas pressure, differential pressure across the orifice, silicon diode temperature sensors on the copper block, and a CCD camera with microscope lens to view the $\sim 25$ - to $100-\mu \mathrm{m}$ droplets through one of the windows. Parameters affecting system operation include the temperature of the droplet generator, the background helium pressure in the vacuum chamber, and the orifice differential pressure. An acceptable operating point for drops-on-demand is at a temperature of $172-173 \mathrm{~K}$ on the droplet generator, $1000-1025$ torr of helium in the vacuum tank, and close to zero pressure differential across the droplet generator orifice. The operating point once achieved is stable; a recent run was made producing $\sim 50-\mu \mathrm{m}$ diam drops at $30 \mathrm{~Hz}$ for $\sim 42$ min (about 75,000 drops). Drop terminal velocities are in all cases $<1 \mathrm{~m} / \mathrm{s}$, and Reynolds numbers are $<1$.

\section{CARBON DIOXIDE PELLETS FOR SURFACE CONDITIONING}

ORNL has developed a technology that uses cryogenic pellets for surface conditioning to replace solvent-intensive and sandblast technology. An ORNL centrifuge driver, which accelerates frozen $\mathrm{CO}_{2}$ (or argon) pellets to high speeds, has potential as a high-performance, energy efficient, and environmentally friendly means for surface cleaning and paint removal. In comparison to the more conventional compressed air "sandblast" pellet accelerators, the ORNL centrifugal accelerator system can achieve higher pellet speeds, has precise speed control, and is more energy efficient. Furthermore, the use of frozen $\mathrm{CO}_{2}$ pellets instead of conventional metal, plastic, sand, or other abrasive materials that remain solid at room temperature, minimizes the waste stream. This apparatus has been used to demonstrate cleaning of various surfaces, including removal of 
paint, oxide coatings, metal coatings, organic coatings, and oil and grease coatings from a variety of surfaces. In the centrifuge acceleration technique, pellets are loaded onto a spinning arbor (accelerator wheel) that accelerates them and then slings them off its outer edge at high speeds. The centrifuge accelerator concept is illustrated in Fig. 5. The arbor with a diameter of $0.35 \mathrm{~m}$ is spun using a brushless, $11 \mathrm{~kW}$ de motor. The motor and arbor are designed to be mounted on a robotic apparatus that can move the device over the desired surface region at a controlled scan rate. Experimental results have shown that the unit will deliver $\mathrm{CO}_{2}$ pellets at feed rates as high as $450 \mathrm{~kg} / \mathrm{h}$ with pellet velocities of $350 \mathrm{~m} / \mathrm{s}$. The pellets are distributed over a swath about $0.13 \mathrm{~m}$ wide as it is scanned across a surface. In an initial test, epoxy-based paint was removed from aluminum panels at a rate of about $6.7 \mathrm{~m}^{2} / \mathrm{h}$ with the panels at room temperature. By raising the panel temperature to about $100^{\circ} \mathrm{C}$, paint stripping rates greater than $22 \mathrm{~m}^{2} / \mathrm{h}$ are possible.

\section{METHANE DROPS FOR USE IN AN ADVANCED COLD NEUTRON MODERATOR}

A variety of cold moderators have been developed and installed in pulsed and continuous, reactor and accelerator-based, neutron sources for the production of cold neutrons. For cold moderators, the principal material choices have been liquid $\mathrm{H}_{2}$, liquid $\mathrm{D}_{2}$, solid $\mathrm{D}_{2} \mathrm{O}$, liquid $\mathrm{CH}_{4}$, and solid $\mathrm{CH}_{4}$. The technology of liquid $\mathrm{H}_{2}$ and $\mathrm{D}_{2}$ is well established. Methane has a higher density of protons than all moderators listed except ammonia; solid methane has an almost $70 \%$ larger proton density than liquid hydrogen. This accounts, in part, for its superior performance as a moderator; the other key factor being a high density of internal rotational modes, which are nearly free even in the lowtemperature solid. Liquid methane can be produced with liquid nitrogen cooling and solid methane by cooling with liquid or supercritical hydrogen or cold gaseous helium.

During this decade, this potential has resulted in installation of liquid and/or solid methane moderators at several neutron science facilities. Radiation-induced effects, however, have limited the ability of solid methane moderators to be used in high-power neutron sources. The practical effects of irradiation of methane are the production of hydrogen and the formation of solid hydrocarbon deposits in the moderator vessel. Solid methane moderators used to date are monolithic blocks that can only be operated for limited periods before the radiation-induced reactions described above cause swelling of the containment vessel and production of oily and tar-like polymerization products. The amount of beam power a solid moderator of this type can handle is also limited due to the low thermal conductivity of solid methane that limits the ability to remove the heat generated.

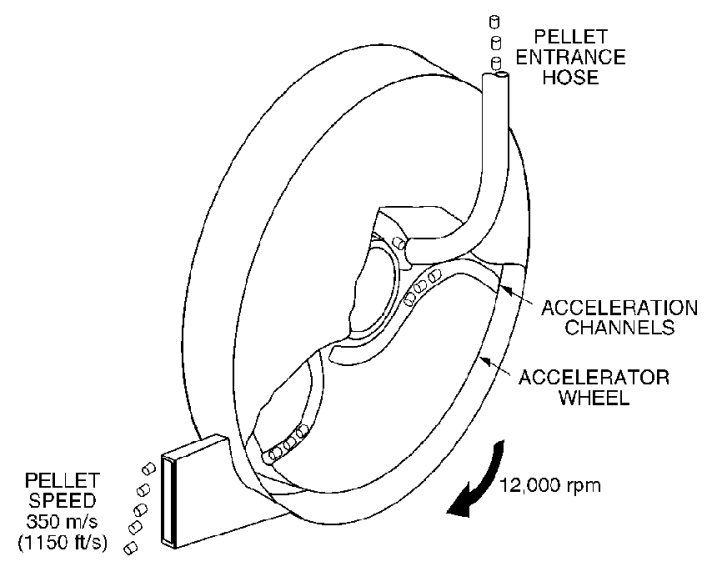

Figure 5. ORNL centrifuge accelerator used for surface conditioning. 
ORNL has proposed an innovative solid methane moderator system capable of operating at power levels up to $1 \mathrm{MW}$ on target. This would yield a flux of low-energy (1- to $10-\mathrm{meV}$ ) neutrons three to four times as intense as that from liquid hydrogen moderators. The basic concept is to use millimeter-size, solid methane spheres surrounded by liquid or supercritical hydrogen at $20 \mathrm{~K}$ (Ref. 7). The hydrogen maintains the methane spheres at $20 \mathrm{~K}$ and is also used to transport the spheres to the active moderator region.

In 1996 an initial scoping experiment was conducted. The goal was to evaluate the feasibility of creating and maintaining a fluidized bed of discrete methane spheres for a cold neutron source. This brief experiment involved the production of $\sim 1$ - to 2-mm-diam methane droplets and the subsequent study of their collective behavior in a research dewar containing liquid hydrogen. Methane gas was fed into a copper droplet generator where liquid methane was produced in the $90-100 \mathrm{~K}$ reservoir. The liquid was then formed into 1to 2-mm droplets via a magnetostrictive plunger action on a diaphragm. The drops fell through the transfer line to the first floor station, where the partially solid drops were collected in the bottom of the research cryostat. Windows on the cryostat allowed observation of the drops and provided insight on their collective behavior in a background bath of liquid hydrogen $\left(\sim 50 \mathrm{~cm}^{3}\right)$. Most of the experiment goals were achieved:

- The methane droplet generator, operating at $92 \mathrm{~K}$ with liquid nitrogen cooling, produced consistent, spherical, methane droplets of diameter $\sim 1 \mathrm{~mm}$ at repetition rates from 1-100 Hz. In the final run, over 300,000 drops were generated at $30 \mathrm{~Hz}$ for $3 \mathrm{~h}$.

- The research cryostat located some $9 \mathrm{~m}$ below the droplet generator produced reliable pools of liquid hydrogen (at 18-19 K), which were used as a reservoir for accumulation of the vertically falling methane droplets.

- The solubility of methane in liquid hydrogen was measured at $<1 \mathrm{ppm}$, which was the limit of sensitivity of the measurement.

This brief test campaign showed that continuous production of consistent drops was indeed feasible. However, it proved difficult to fully freeze the drops during their freefall to the liquid hydrogen in the research dewar, which limited the quality of the fluid-solid bed. A more direct solid pellet production method is probably required, such as extrusion or molding solids via batch processing.

\section{ACKNOWLEDGMENT}

Research at Oak Ridge National Laboratory was sponsored by the U.S. Department of Energy under contract DE-AC05-96OR22464 with Lockheed Martin Energy Research Corp.

\section{REFERENCES}

1. S. L. Milora et al., Review paper: pellet fuelling, Nucl. Fusion 35:657 (1995).

2. S. K. Combs, Pellet injection technology, Rev. Sci. Instrum. 67:1679 (1993).

3. P. W. Fisher and M. J. Gouge, Extrusion of tritium and DT pellets for ITER fueling, Fusion Technology 30:845 (1996).

4. M. J. Gouge, Fueling of ITER-scale fusion plasmas, Fusion Technology 34:435 (1998).

5. D. J. Nagel, in: "VLSI Electronics," M. Brown, ed., Academic, New York (1984), Vol. 8.

6. M. J. Gouge and P. W. Fisher, A cryogenic xenon droplet generator for use in a compact laser plasma X-ray source, Rev. Sci. Instrum. 68:2158 (1997).

7. A. T. Lucas, A combined $\mathrm{H}_{2} / \mathrm{CH}_{4}$ moderator for a short pulsed neutron source, "Proceedings of ICANS-X Conference, Los Alamos, New Mexico, October 3-7, 1988," D. K. Dyer, ed., Institute of Physics Conference Series No. 97, Institute of Physics, Bristol and New York (1989). 